\title{
Radiation induced mutagen sensitivity and chlorophyll mutation frequency on sesame seeds
}

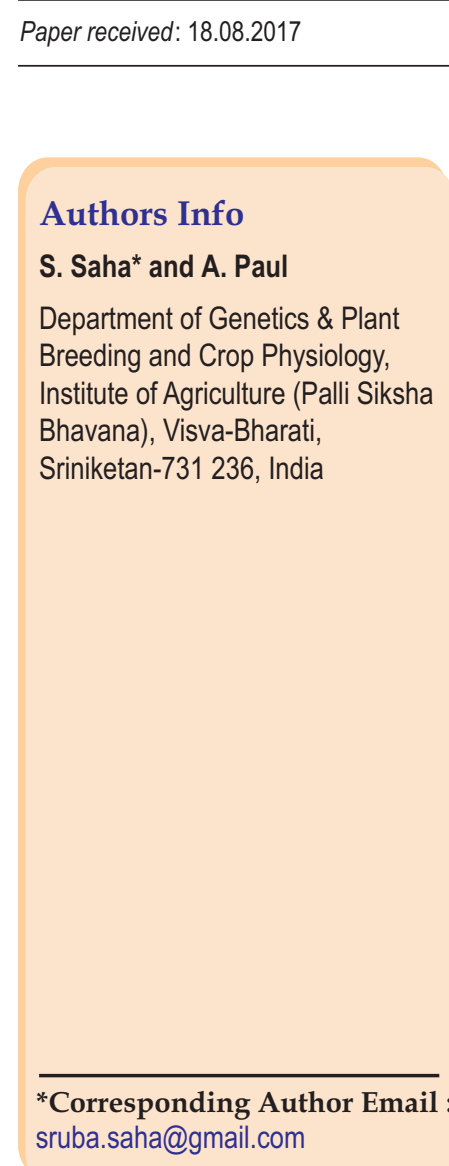

Edited by
Dr. R. C. Dalela

Reviewed by

Dr. R. B. Raizada

Revised received: 21.12 .2017

Re-revised received: 14.06 .2018

Accepted: 19.07 .2018

\section{Abstract}

Aim: Effect of gamma irradiation on genomic disorder in sesame are scanty. The present study was undertaken to evaluate the mutagenic effects of gamma rays on different parameters in two popular cultivars of sesame, Roma and Tilottama.

Methodology: Seeds of these two cultivars were irradiated with five doses of gamma rays: $(250,300,350$, 400 , and $450 \mathrm{~Gy}$ ) at BARC, Trombay and were sown (along with the un-irradiated control) during March 2015 in a split plot design with 3 replications keeping row to row and plant to plant distance at $30 \mathrm{~cm}$ and 10 $\mathrm{cm}$, respectively to determine mutagen sensitivity with regard to pollen fertility $(\%)$, germination (\%) and seedling height $(\mathrm{cm})$, root-shoot length $(\mathrm{cm})$, plant survival $(\%)$ at maturity in $\mathrm{M}_{1}$ generation. To study mutability, four to five capsules from each $\mathrm{M}_{1}$ plants in all the treatments were collected separately to give rise the $\mathrm{M}_{2}$ generation. Individual plant progeny rows were sown in $\mathrm{M}_{2}$ during March 2016. ID ${ }_{50}$ was determined by probit analysis for germination, shoot-root length and plant survival. Since the dose requirement for pollen fertility is very high, $I D_{30}$ was calculated instead of $I D_{50}$ value.

Results: It was observed that with increasing doses of gamma rays, the response of all characters decreased significantly and followed a linear relationship in both varieties. The root system was more profound to gamma rays than the shoot. Chlorophyll mutations showed independent response to different doses of gamma rays as they occurred in random. The mutability of genotype Roma induced with different doses of gamma rays was higher than that of Tilottama.

Interpretation: Due to saturation in the mutational actions, response of characters decreased with increase in gamma ray doses but magnitudes of effect differed between genotypes. The cultivar Tilottama was found to be more sensitive than Roma.

Key words: Gamma rays, Inhibitory dose, Mutability, Mutagen sensitivity, Sesame

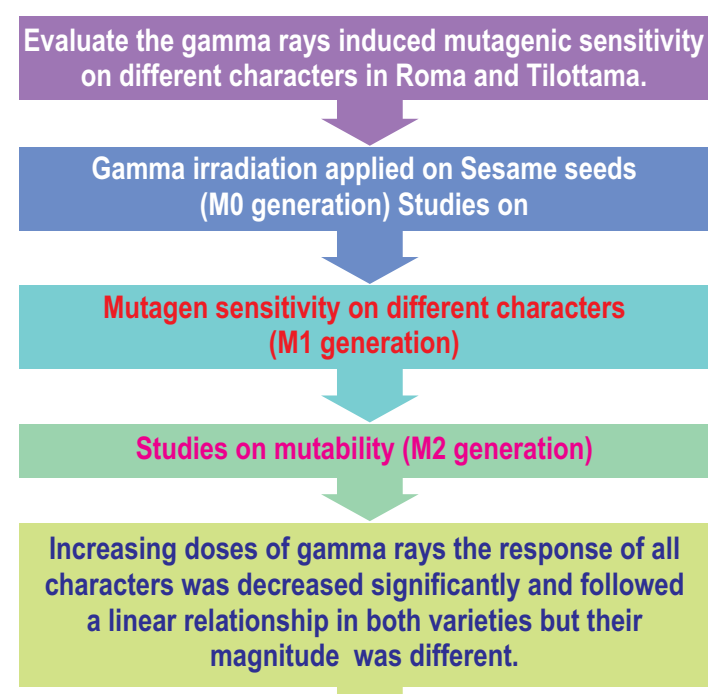

The mutability of the genotype Roma induced with different doses of gamma rays was higher than that of Tilottama

How to cite : Saha, S. and A. Paul: Radiation induced mutagen sensitivity and chlorophyll mutation frequency on sesame seeds. J. Environ. Biol., 40, 252-257 (2019). 


\section{Introduction}

Sesame (Sesamum indicum L.; $2 n=26$ ) also known as til (in Hindi) is probably the oldest and traditional oilseed crop known to man and is valued for its high-quality seed oil (Anilakumar et al., 2010). It is the fifth most important edible oilseed crop in India after groundnut, rapeseed-mustard, sunflower and soybean, although the productivity of sesame stands alarmingly poor comparing to other oil yielding crops. As a quality oil seed crop, it can reduce the shortage of other edible oil production in the country, if the production and productivity are enhanced. Sesame is a pre-kharif crop in India and the agricultural importance is increasing as a large part of peninsular India is becoming prone to drought. Mutation breeding in crop improvement program for developing varieties with desirable agro-morphic traits is common in other crops but rarely implemented for sesame in India (Begum and Dasgupta, 2015).

Induced mutagenesis has been effectively employed to improve the productivity as well as to generate a good amount of variability in morphological and physiological characters by inducing new plant ideotypes. Mutation means a sudden heritable change in the genetic material at gene or chromosome level (Chahal and Gosal, 2002). They may be caused by an error during cell division or by exposure to DNA-damaging agents or mutagens in the environment. A wide range of characters which have been improved through induced mutation breeding includes plant architecture, yield, flowering and maturity duration, quality and tolerance to biotic and abiotic stresses. About $89 \%$ of mutant varieties have been developed using physical mutagens such as $\mathrm{X}$-rays, gamma rays, thermal and fast neutrons, whereas with gamma rays alone accounting for the development of $60 \%$ of the mutant varieties (Kharkwal, 2000).

The two most commonly used breeding approaches in sesame are selection (mostly from local landraces) and pedigree; introduction, backcross, and induced mutations are less common (Ashri, 2001). Until recently most of the released sesame varieties in countries such as China, India and the Republic of Korea was the product of selection and pedigree breeding. A major constraint in this approach was the lack of sufficient genetic variation within the existing germplasm collections, especially for traits such as earliness, ideal plant architecture, resistance to various diseases and insect-pests and seed retention (Ashri, 2001). This is where mutation techniques can offer a possible solution. Mutations induced by radiation or chemicals provide variation in plant structure and function from which breeders can select plants having useful traits. Gustafsson (1947) advocated that mutation approach be superior to other methods of crop improvement for generation of genetic variability. The most important aspects of mutation breeding is the quick rectification of defects in varieties and advanced breeding lines, induction of polygenic mutations and development of ideotypes for various agro-climatic conditions. In past, researchers have worked to improve the agro-morphic traits such as determinate growth habits (Cagirgan, 2006), early matured (Uzun, 2006), larger seed size and seed colour (Hoballah, 2001) and resistance to Fusarium wilt (Silme, 2010) in sesame.

To induce variability for efficient plant breeding, systematic study of mutagenic sensitivity and/or mutability of various crop plants and different genotypes within a crop are essential (Brock, 1971). Although, studies have been made on the biological effects of radiations and relative mutagens sensitivity in mung bean (Ignacimuthu and Babu, 1989), urd bean (Singh et al., 1999), chickpea (Kharkwal, 1998) and field pea (Paul and Mondal, 2012), however, such reports are limited in sesame. Therefore, in the present investigation, an attempt was made to study the mutagen sensitivity in the two high yielding genotypes of sesame. The similarities or differences between genotypes with regards to radio-sensitivity, $I D_{50}$ and relationship between mutagen sensitivity and mutability following gamma treatment to those sesame varieties in $M_{1}$ and $M_{2}$ generation was also studied.

\section{Materials and Methods}

The study was carried out at Agriculture Farm of Palli Siksha Bhavana (Institute of Agriculture), Visva-Bharati, Sriniketan in pre-kharifseason of 2015-16.

Plant materials and their basic characteristics : "Roma": brown, rough, glossy seeds ( 1000 seed weight $\sim 3.5 \mathrm{gm}$ ), medium in size, maturity (92-100 days) and "Tilottama": black, rough, dull seeds ( 1000 seed weight $\sim 3.1 \mathrm{gm}$ ), medium in sizes, maturity (100-110 days) genotypes of sesame (till) were procured from Pulse and Oilseed Research Centre, Baharampur, West Bengal, India for the present study.

Gamma irradiation : 10,000 dry, uniform and healthy seeds of these two genotypes of sesame were irradiated using ${ }^{60} \mathrm{Co}$ (Cobalt 60) gamma source (Gamma Chamber 900) with different doses (250, 300, 350, 400, $450 \mathrm{~Gy}$ ) of gamma rays at the Bhabha Atomic Research Centre (BARC), Trombay, India.

Field experiments : Irradiated seeds $\left(\mathrm{M}_{0}\right)$ along with the controls (un-irradiated) were sown in the field (treatment and variety wise) in a Randomized Block Design with three replications during prekharif season of 2015. Four to five capsules of each $M_{1}$ plants against all the treatments were collected separately to raise the $\mathrm{M}_{2}$ generation. Data were taken in the field as well as in laboratory on germination (\%), pollen fertility (\%), seedling height (cm), rootshoot length $(\mathrm{cm})$, survival $(\%)$ in $\mathrm{M}_{1}$ generation and chlorophyll mutation in $\mathrm{M}_{2}$ generation.

Data on germination in the field on each treatment were recorded after 6 to 7 days of sowing and germination percentage was calculated. Seedling height $(\mathrm{cm})$ was also recorded in field after 15 days of sowing. Treated and untreated seeds were 
spread over moist germinating paper in petri plates with three replications for each treatment and observed the shoot and root length $(\mathrm{cm})$ of treated seeds under laboratory conditions.

For recording pollen fertility, flower buds were collected from randomly selected 10 plants in each replication under each dose. Study on the pollen fertility was done by using $2 \%$ acetocarmin solution. Pollen grains stained were considered fertile while deformed or poorly stained or translucent ones were sterile. Pollen counts were taken under the stereoscopic microscope from five randomly selected plots and fertility percentage was calculated. Individual plant to progeny rows were grown in $\mathrm{M}_{2}$ generation (pre-kharif, 2016) keeping row to row and plant to plant distance at $30 \mathrm{~cm}$ and $15 \mathrm{~cm}$, respectively.

Chlorophyll mutation frequency : Observations were taken on various types of chlorophyll mutation to study the mutagenic effect of different doses in $\mathrm{M}_{2}$ generation. Chlorophyll mutants were identified tagged and counted just after germination. The process continued for a few days until the completion of germination. At the same time, normal looking plant population was also counted and recorded dose wise in each variety to estimate the chlorophyll frequency. Frequency of chlorophyll mutation was analyzed as per the method described by Konzak et al. (1972).
Statistical analyses : The average was assessed from regression line, $Y=a+b X(Y=$ respone; $X=$ dose) by using linear regression analysis. $I_{50}$ value using Probit Analysis (Finney, 1971) after Abbott's (1925) correction of each treatment with respect to control response. Level of sensitivity of both the genotypes was also compared with the help of independent sample t-test. Frequency of chlorophyll mutation was analyzed statistically. All statistical analyses were carried out by SPSS 20.0 and Microsoft's Excel 2007.

\section{Results and Discussion}

It was clearly understood from the steepness of the regression slopes that the reduction percentage of germination increased significantly with increasing dose of gamma rays and followed a linear relationship in Roma (Adjusted $R^{2}=0.995$, $p<0.001$ ) as well as in Tilottama (Adjusted $R^{2}=0.991, p<0.001$ ) (Fig. 1a). Reduction of seed germination (\%) at high doses could be due to the damage of chromosomes and subsequent mitotic activities which depends upon the doses used (Dutta, 2009). Increasing dose of gamma rays increased the reduction percentage of seedling height $(\mathrm{cm})$ with respect to control (untreated seeds), significantly in Roma (Adjusted $R^{2}=0.93$, $p<0.001$ ) and in Tilottama (Adjusted $R^{2}=0.98, p<0.001$ ) (Fig. 1b). The regression slopes revealed reduction of seedling height in

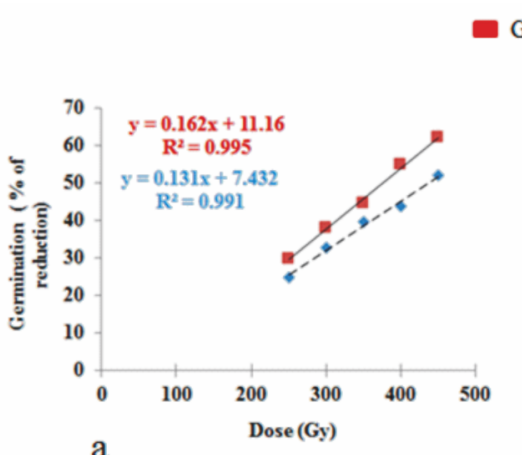

a
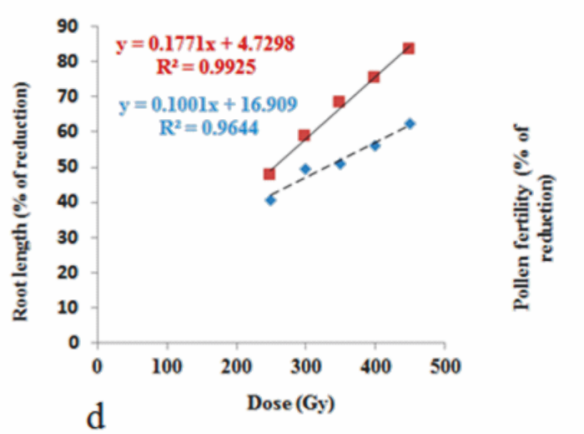

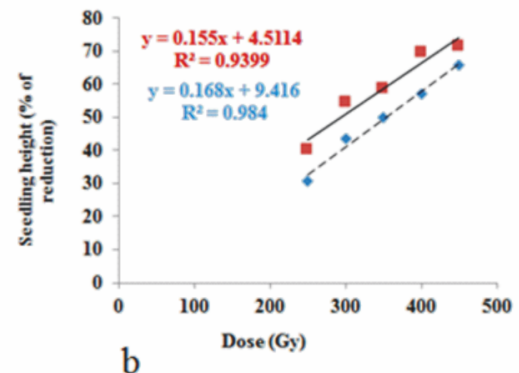

b

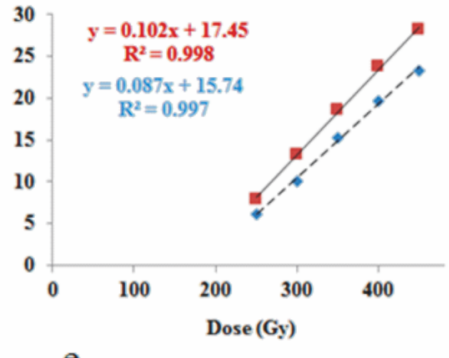

e
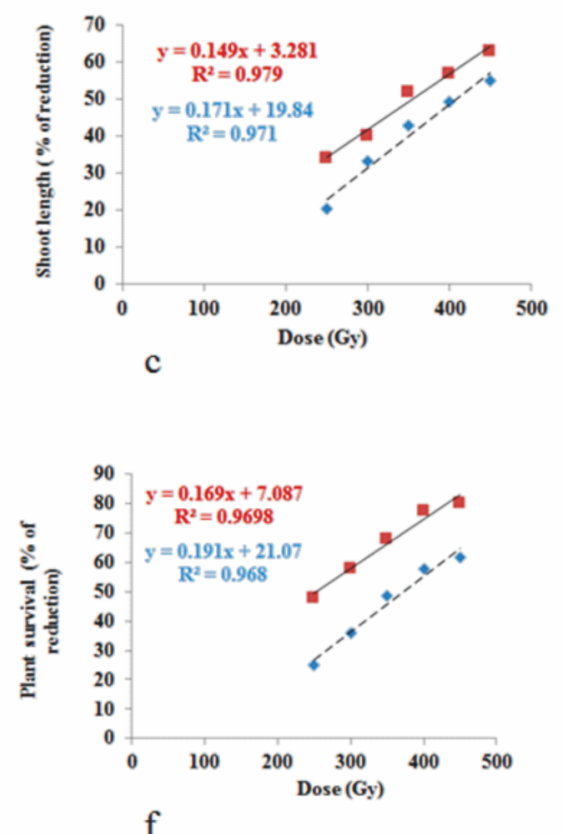

Fig. 1 : Influence of Gamma ray irradiation on germination (a), seedling height (b), shoot length (c), root length (d), pollen fertility (e) and plant survival (f). 
Table 1 : Median inhibition dose and radio-sensitivity of two sesame genotypes for different quantitative traits in $\mathrm{M}_{1}$ generation

\begin{tabular}{llll}
\hline Quantitative traits & \multicolumn{2}{c}{ Genotypes } & Radio-sensitivity \\
\cline { 2 - 3 } & Roma & Tilottama & \\
\hline Germination & $440.15 \mathrm{~Gy}(393.97-558.84))$ & $370.68 \mathrm{~Gy}(342.74-410.99)$ & Tilottama>Roma \\
Rootlength $(\mathrm{cm})$ & $323.66 \mathrm{~Gy}(262.88-371.52)$ & $262.03 \mathrm{~Gy}(226.26-285.63)$ & Tilottama>Roma \\
Shoot length $(\mathrm{cm})$ & $405.34 \mathrm{~Gy}(375.67-456.56)$ & $349.44 \mathrm{~Gy}(319.24-385.26)$ & Tilottama>Roma \\
Pollen fertility* & $407.85 \mathrm{~Gy}(369.08-491.40)$ & $367.90 \mathrm{~Gy}(333.43-412.51)$ & Tilottama>Roma \\
Plant survival & $366.32 \mathrm{~Gy}(343.14-396.34)$ & $256.35 \mathrm{~Gy}(215.23-282.24)$ & Tilottama>Roma \\
Seedling Height $(\mathrm{cm})$ & $346.70 \mathrm{~Gy}(320.11-376.60)$ & $290.71 \mathrm{~Gy}(252.38-316.57)$ & Tilottama>Roma \\
\hline
\end{tabular}

${ }^{*} \mathrm{D}_{30}(\mathrm{~Gy})$ value calculated; Data in parentheses indicate fiducial low and up values (Gy)

Table 2: Frequency (\%) of three types of chlorophyll mutations between two genotypes

\begin{tabular}{llllll}
\hline Variety & Mutagen treatment & \multicolumn{3}{c}{ \% of chlorophyll mutation } & Total frequency (\%) \\
\cline { 3 - 5 } & & Albina & Chlorina & Xantha & \\
\hline Roma & $250 \mathrm{~Gy}$ & 0.24 & 0.24 & 0.28 & 0.77 \\
& $300 \mathrm{~Gy}$ & 0.32 & 0.24 & 0.30 & 0.87 \\
& $350 \mathrm{~Gy}$ & 0.30 & 0.44 & 0.51 & 1.25 \\
\multirow{4}{*}{ Tilottama } & $400 \mathrm{~Gy}$ & 0.63 & 0.36 & 0.22 & 1.22 \\
& $450 \mathrm{~Gy}$ & 0.60 & 0.35 & 0.49 & 1.43 \\
& $250 \mathrm{~Gy}$ & 0.24 & 0.24 & 0.27 & 0.76 \\
& $300 \mathrm{~Gy}$ & 0.37 & 0.47 & 0.43 & 1.28 \\
& $350 \mathrm{~Gy}$ & 0.45 & 0.39 & 0.47 & 1.30 \\
& $400 \mathrm{~Gy}$ & 0.51 & 0.32 & 0.47 & 0.93 \\
\hline
\end{tabular}

Tilottama genotype was higher than their Roma counterparts, however, as dose increased Roma became more sensitive.

Different mutagenic treatments influenced the vigour of seedling height. In Fig. 1c and $1 \mathrm{~d}$ both genotypes showed almost parallel regression line, although, the shoot length was more affected in higher dose in Tilottama (Adjusted $R^{2}=0.97, p<0.001$ ) followed by Roma (Adjusted $R^{2}=0.97, p<0.001$ ). In the case of both varieties, among the treatments, 450 Gy recorded significantly highest reduction of shoot length followed by other treatment. Both varieties exposed gradual reduction of root length with an increase of doses. However, the higher regression slope had indicated that the genotype Tilottama (Adjusted $R^{2}=0.99$, $P<0.001)$ exhibited a greater reduction in root growth than the genotype Roma (Adjusted $R^{2}=0.96, P<0.001$ ). Seedling height along with root and shoot length is widely used as an index in determining the biological effects of various physical and chemical mutagens in $\mathrm{M}_{1}$ (Konzak et al., 1972). According to Wi et al. (2007), low dose of irradiation induces growth stimulation by changing the hormonal signalling network in plant cells. In contrast, high-dose irradiation that caused growth inhibition has been recognized to cell cycle and various damages in the entire genome (Perussa et al., 2003). In this study, the root growth of Tilottama remarkably affected the shoot elongation through irradiation. This trend confirms the results from recent studies in Withamnia somnifera (Bhosala and Mose, 2014), in Lentill (Rana et al., 2014) and tomato (Sikdar et al., 2013). The biological processes behind this trend can be attributed to the higher inhibition of cell division in root cells by gamma irradiation, which showed less impact on the shoot elongation.

With the increase in gamma ray doses, reduction in pollen fertility (measured in \%) decreased significantly and followed a linear relationship in Roma (Adjusted $R^{2}=0.99, p<0.001$ ), as well as in Tilottama (Adjusted $R^{2}=0.92, p<0.001$ ) (Fig.1e). The sensitivity of gamma radiation in reduction of survival rate $(\%)$ was evaluated in both the genotypes, Roma (Adjusted $R^{2}=0.97$, $p<0.001$ ) and Tilottama (Adjusted $R^{2}=0.97, p<0.001$ ) (Fig.1f). The responses were higher in Tilottama than Roma due to higher and steeper slope of regression line. Kiong et al. (2008) observed that survival of plants to maturity depends on the nature and extent of chromosomal damage. Increasing frequency of chromosomal damage with radiation dose may be responsible for lesser capacity to germinate and reduction in plant growth and survival.

Inhibitory dose $\left(\mathrm{ID}_{50}\right.$ and $\left.\mathrm{ID}_{30}\right)$ along with their fiducial values (Table 1) for different variables studied in $M_{1}$ generation 
revealed that reduction in $50 \%$ germination $\left(\mathrm{ID}_{50}\right)$ occurred at 440.15 Gy in Roma, whereas slightly lower dose (370.68 Gy) was recorded for the genotype Tilottama. $I D_{50}$ values of shoot and root length was $405.34 \mathrm{~Gy}$ in Roma, 349.44 Gy in Tilottama and 323.66 Gy for Roma, 262.03 Gy for Tilottama, respectively. In the case of plant survival at maturity, $I_{50}$ value of Tilottama was 256.35 Gy, whereas, the genotype Roma exhibited higher reduction in $366.32 \mathrm{~Gy}$. Another immediate effect of irradiation in $M_{1}$ generation was also observed through reduction in pollen fertility for which $\mathrm{ID}_{30}$ was $407.85 \mathrm{~Gy}$ and $367.90 \mathrm{~Gy}$ for Roma and Tilottama, respectively.

The inhibitory dose is one of the important factors to predict radiosensitivity level in plants (Kumar et al., 2013). It quantifies a dose where $50 \%$ of seeds can survive, germinate and expose to their normal growth. In this study, in of case pollen fertility, $I D_{30}$ value was calculated instead of $I_{50}$. The reason behind that was more than $450 \mathrm{~Gy}$ of gamma irradiation were required to inhibit $50 \%$ pollen fertility for both genotypes, which crosses the limit of the irradiation dose. This indicates that treated doses in this experiment were safe for the particular parameter.

Three types of chlorophyll mutations such as: chlorina, albina, xantha were recorded in different frequencies, chlorina being in the highest order followed by xantha, then albina (Table 2). Chlorophyll mutations showed an independent response to different doses of gamma rays as they occurred at random, and the mutability of genotype Tilottama induced with different doses of gamma rays was lower than that of Roma. In both genotypes, the frequency of chlorophyll mutants increased with an increase in gamma ray doses. The highest frequency of total three types was recorded at 450 Gy in Roma (1.43).

The frequency of chlorophyll mutation serves as a good index to determine different doses of mutagens. Further, induced chlorophyll variations serve as diagnostic markers for available mutation in the irradiated population. According to Ali et al. (2016) photosynthetic complex is altered by radiations. Ionizing radiations decrease the capabilities of photosynthetic apparatus by damaging the photosystem (Angelini et al., 2000). Pre-kharif and summer season crops like sesame growing under high light intensities: high doses of gamma radiations could damage the photosynthetic pigments that reduced photosynthetic capabilities of plants. A high dose of gamma rays up to $500 \mathrm{~Gy}$ decreases chlorophyll content by $80.91 \%$ and decreases the organized pattern of grana and stroma thylakoid (Alikamanoglu et al., 2011).

In the present study, two genotypes of sesame proved to be highly responsive to different doses of gamma rays but based on all studied characters, the magnitude of sensitivity was always higher in the genotype Tilottama than Roma. The effect of irradiation on different quantitative trait mainly depends upon the applied doses and genetic constitution of experimental materials. Higher dose causes more reduction. Similar findings were observed by Selem et al. (2018) in sesame and Hong et al. (2018) in wheat. Overall, this study indicated mutation creates variation in the experimented population.

\section{Acknowledgments}

This work was supported by the Bhaba Atomic Research Centre (BARC), Trombay, India by the funding [No. 35/14/09/2014-BRNS] received from the Department of Atomic Energy, Board of Research in Nuclear Sciences, Government of India (DAE-BRNS, Govt. of India). We express our heartfelt thanks to Dr. Palash Mondal of the Institute of Agriculture, VisvaBharati, for sharing his knowledge during the preparation of this manuscript.

\section{References}

Abbott, W.S.: A method for computing the effectiveness of an insecticide. J.Econ. Ent. 18, 265-67 (1925).

Ali, H., Z. Ghori, S. Sheikh and A. Gul: Effects of gamma radiation on crop production. Springer International Publishing Switzerland 2016 (Ed. K.R. Hakeem). Crop Produc. Global Environ. Issu., 2 (2016). DOI:10.1007/978-3-319-23162-4.

Alikamanoglu, S. and A. Sen: Stimulation of growth and some biochemical parameters by magnetic field in wheat (Tritium aestivum L.) tissue cultures. African J. Biotech., 10, 10957-10963 (2011).

Angelini, G., P. Ragni, D. Esposito, P. Giardi, M. Pompili, R. Moscardelli and M. Giardi:Adevice to study the effect of space radiation on photosynthetic organisms. Phys. Med., 17, 267-268 (2000).

Anilakumar, K.R., A. Pal, F. Khanum and A.S. Bawa: Nutritional, medicinal and industrial uses of sesame (Sesamum indicum L.) seeds - An overview. Agric.Conspec. Sci., 75, 159-168 (2010).

Ashri, A.: Sesame (Sesamum indicum L.). In: Genetic Resources, Chromosome Engineering, and Crop Improvement, Oilseed Crops (Ed.: R.J. Singh). CRC Press, BocaRaton, FL, USA, 4, 231 289 (2001).

Begum, T. and T. Dasgupta: Amelioration of seed yield, oil content and oil quality through induced mutagenesis in sesame (Sesamum indicum L.). Bangladesh J. Bot., 44, 15-22 (2015).

Bhosale, R.S. and A.D. More: Effect of gamma radiation on seed germination, seedling height and seedling injury in Withania somnifera (L.) Dunal. Int. J. Life Sci., 2,226-228 (2014).

Brock, R.D.: The role of induced mutations in plant improvement. Rad. Bot., 11, 181-96 (1971).

Cagirgan, M.I.: Selection and morphological characterization of induced determinate mutants in sesame. Field Crops Res., 96, 19-24 (2006).

Chahal, G.S. and S.S. Gosal: Principles and Procedures of Plant Breeding. Oxford, Alpha Science International Ltd., pp. 399-412 (2002).

Datta, S.K.: Areport on 36 years of practical work on crop imp rovement through induces mutagenesis. In: Induced plant mutations in the genomics era (Ed.: Q.Y. Shu). Food and Agriculture Organization of the United Nations, Rome, pp. 253-256 (2009).

Finney, D.J.: Probit Analysis. $3^{\text {rd }}$ Edn., Cambridge University Press, U.K. p. 333 (1971).

Gustafsson, A.: Mutation in agricultural plants. Heriditas, 33, 1-100 (1947). 
Hoballah, A.A.: Selection and agronomic evaluation of induced mutant lines of sesame. In: Sesame improvement by induced mutations, IAEA-TECDOC-1195. IAEA, Vienna, pp. 137-150 (2001).

Hong, J.M., D.Y. Kim, J.W. Ahn, S.Y. Kang, Y.W. Seo and J.B. Kim: Comparison of radiosensitivity response to acute and chronic gamma irradiation in colored wheat. Genet. Mol. Bio., 41, 611-623 (2018).

Kiong, A., A. Ling Pick, S.H. Grace Lai and A.R. Harun: Physiological responses of Orthosiphon stamineus plantlets to gamma irradiation. Am. Eurasian J. Sustain. Agric., 2, 135-149 (2008).

Kumar, D.P., A. Chaturvedi, M. Sreedhar, M. Aparna, P.V. Babu and R.K. Singhal: Gamma radiosensitivity study on rice (Oryza sativa L.). Asian J. Plant Sci. Res., 3, 54-68 (2013).

Kharkwal, M.C.: Induced mutations in chickpea (Cicer arietinum) V. Evaluation of micromutations. Indian J. Genet. Plant Breed., 61, 115-24 (1998).

Kharkwal, M.C.: Induced mutations in chickpea (Cicer arietinum L.) IV. Types of macromutations induced. Indian. J. Genet. Plant Breed., 60, 305-320 (2000)

Preussa, S.B. and A.B. Britta: A DNA-damage induced cell cycle checkpoint in Arabidopsis. Genetics, 164, 323-334 (2003).

Paul, A. and P. Mondal: Effect of gamma radiation on mutagen sensitivity and mutability in field pea (Pisum sativum L.). J. Crop Weed., 8 , 132-136 (2012).
Rana, A. and I.S. Solanki: Ethyl methane sulphonate induced genetic variability and heritability in macrosperma and microsperma lentils. J. Environ. Biol., 36, 1119-1123(2015).

Selem E.E., R.E.A. Hamed, H.A. Kamel and H.S. Hegazy: Physiological and biochemical response of gamma irradiated Sesamum indicum L. seeds grown in heavy metal contaminated soil. Bios. Res., 15, 1063-1072 (2018).

Silme, R.S. and M.I. Cagirgan: Screening for resistance to Fusarium wilt in induced mutants and world collectionof sesame under intensive management. Turk. J. Field Crops., 15, 89-93 (2010).

Sikder, S., P. Biswas, P. Hazra, S.A. Khtar, A. Chattopadhyay, A.M. Badigannavar and S.F. D'Souza: Induction of mutation in tomato (Solanum lycopersicum L.) bygamma irradiation and EMS. Indian J. Genet. Plant Breed., 73, 392-399 (2013).

Singh, V.P., M. Singh and J.P. Pal: Mutagenic effects of gamma rays and EMS frequency and spectrum of chlorophyll and macromutations in urd bean Vigna mungo L. Hepper. Indian J. Genet. Plant Breed., 59, 203-10 (1999).

Uzun, B., S. Ulger and M.I. Cagirgan: Comparison of determinate and indeterminate types of sesame for oil content and fatty acid composition. Turk. J. Agric. For., 26, 269-274 (2002).

Wi, S.G., B.Y. Chung and J.S. Kim: Effects of gamma irradiation on morphological changes and biological responses in plants. Micron, 38, 553-564 (2007). 